\title{
Anti-Aging Medicine and Reproductive Health
}

\author{
Masao Jinno ${ }^{1)}$, Hiroshi Tamura ${ }^{2)}$, Yoshikazu Yonei ${ }^{3)}$ \\ 1) Women's Clinic Jinno \\ 2) Department of Obstetrics and Gynecology, Yamaguchi University Graduate School of Medicine \\ 3) Anti-Aging Medical Research Center, Graduate School of Life and Medical Sciences, Doshisha University
}

\begin{abstract}
Given that ovarian function declines with age, older women occasionally experience health or fertility problems. Functional change is influenced by physical, mental, oxidative, and glycation stresses, which may lead to a decline in the secretion of hormones, such as melatonin, growth hormone/insulin-like growth factor-I, and dehydroepiandrosterone (DHEA). Many different factors influence the degradation of ovarian function, and the proper treatment depends on the accurate identification of each patient's condition. Potential degradation mechanisms include the accumulation of advanced glycation end products (AGEs) by glycation stress, and the activation of receptors for AGEs. Accumulated evidence has suggested that in vitro fertilization success rate can be increased on administration of melatonin and DHEA, or glycation stress therapy, to patients with poor ovarian function. The provision of advanced medical technology to rejuvenate ovarian function should be combined with prophylactic lifestyle guidance, such as that developed by Anti-Aging Medicine, to treat the underlying causes of ovarian functional decline. Here, we review the application of Anti-Aging Medicine to aspects of reproductive medicine.
\end{abstract}

$\boldsymbol{K} \boldsymbol{E} \boldsymbol{Y} \boldsymbol{W O R} \boldsymbol{D S}:$ melatonin, DHEA (dehydroepiandrosterone), IGF-I (insulin-like growth factor-I), growth hormone (GH), AGEs (advanced glycation end products)

\section{Introduction}

Anti-Aging Medicine belongs to a category of preventive medicine that aims to improve the quality of life (QOL) among older persons ${ }^{1}$. In Japan, morbid aging is assessed by a check up (called an 'Anti-Aging dock') that is based on research into aging mechanisms. We believe that Anti-Aging Medicine can promote health and improve QOL for many elderly persons by allowing them to live independently (care prevention), increasing mobility, and reducing cognition disorder and cancer rates. Given that functional degeneration begins in middle age for both genders, treatments derived from Anti-Aging Medicine may also be applicable in these younger populations, with AntiAging coaching and medical treatment helping to improve the health and of these individuals as well as in the elderly.

Ovarian function, which has a profound effect on women's health and QOL, may be improved with Anti-Aging Medicine. The holistic application of Anti-Aging Medicine (from an ovary to the whole body and from the whole body to an ovary) may prevent or ameliorate ovarian functional degradation, a response which would subsequently increase fertilization and implantation rates during in vitro fertilization (IVF) and therefore be applicable to general reproductive medicine.

Such a holistic approach is based on a therapeutic regimen that may include lifestyle guidance, alimentotherapy, ergotherapy, and counseling, as well as medical therapies, such as functional food, nutritional supplements, anti-oxidation, immune response enhancement, hormone replacement, and aesthetic dermatology. However, the success of such therapies depends on the proper identification of risks associated with aging and individual patient needs.

\section{Diagnosis of aging and risk factors}

The first step in Anti-Aging treatment is to assess the functional age and condition of different bodily systems. In Anti-Aging Medicine, an initial medical check, called the AntiAging dock, is conducted to evaluate the condition of bones, blood vessels, muscles, and neural nerves, and to determine hormonal age. This assessment indexes aging, evaluates the whole body balance, and allows subsequent medical treatment to focus on specific tissues or body systems that show significant functional decline ${ }^{1)}$. In addition to evaluation of functional age, the Anti-Aging dock also evaluates other aging risk factors, such as immune function, oxidative, mental, physical, and metabolic (glycation) stress, and lifestyle. 


\section{Evaluation of functional age}

\section{Hormone age}

Given that concentrations of most hormones fall with age, hormone age can be evaluated based on blood hormone levels ${ }^{1)}$.

\section{a. Female hormones}

Estradiol (E2), the most active compound in estrogen, is secreted primarily from follicles in the ovary. Human ovaries contain a fixed number of immature follicles, with one follicle maturing each month and discharging an ovum. On discharge, the follicle secretes E2, thereby producing a periodic change in the E2 blood concentration (Fig. 1) ${ }^{2}$. Ovarian follicles that do not mature are resorbed by atretic follicles.

At birth, the ovaries contain approximately two million primordial oocytes, but roughly 1,000 of these are resorbed every month, leaving approximately 100,000-200,000 oocytes remaining at puberty. About age 50, insufficient ovarian follicle tissue remains to secrete E2, and the periodic secretion of E2 ceases, thereby inducing menopause. Although levels of E2 in blood do not change prior to menopause, secretion stops suddenly after menopause (Fig. 2). This rapid post-menopausal decline in E2 secretion is distinct from the gradual age-related decline observed for other hormones ${ }^{2)}$.

Estrogen plays a central role in reproduction and regulates ovary function, oocyte maturity, and the growth of the endometrial membrane. However, estrogen activity is not limited to the womb and ovaries, as receptors may also be found in the brain, blood vessels, heart, mammary glands, intestinal tract, and bones ${ }^{3)}$. Estrogen deficiency can therefore have a markedly negative influence on several tissues, as well as on sugar and lipid metabolism ${ }^{4}$, climacteric condition, generative functions, vasomotor nerve condition, and bone metabolism ${ }^{5}$.

From the viewpoint of the Anti-Aging of the ovary, one main goal is to prevent decreases in ovum and ovarian follicles. From the viewpoint of general Anti-Aging, however, it is important how to compensate for post-menopausal declines in estrogen secretion by specific treatment, such as hormone replacement therapy. In preventive medicine, the key to AntiAging of the ovary is the prevention of ovarian follicle loss.

Early menopause occurs if the ovarian follicle declines rapidly, and it is diagnosed as premature ovarian failure (POF) if it occurs before age 40. Occasionally iatrogenic factors,

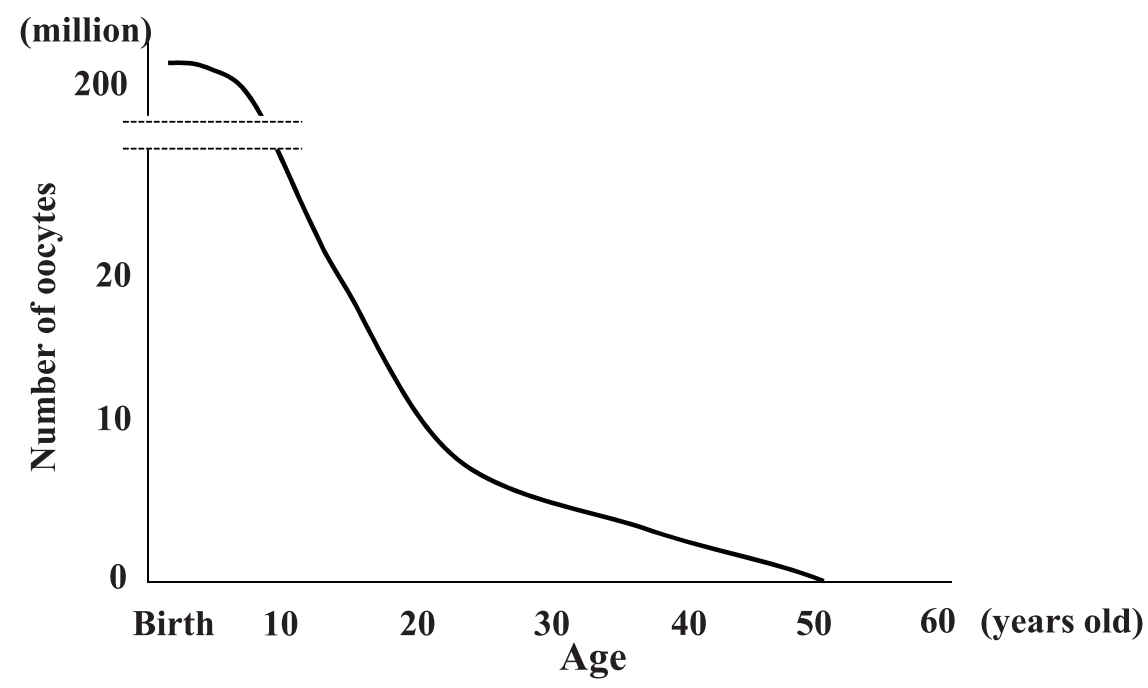

Fig. 1. Change in number of oocytes with age

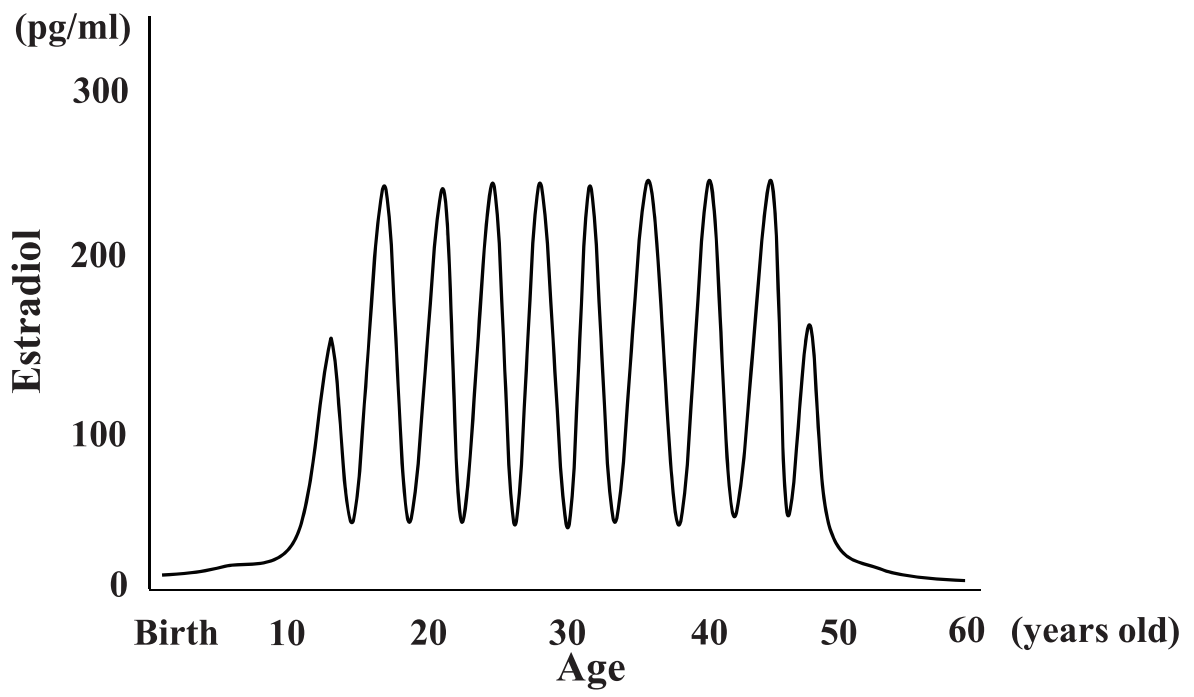

Fig. 2. Change in serum estradiol concentration with age 
such as anti-cancer chemotherapy and radiotherapy 6,7$)$, or autoimmune disease may also trigger precocious menopause. However, many causes of POF are unknown.

The rate of decline in ovarian follicles varies between individuals and may be influenced by factors, such as lifestyle, diet, pregnancy, and inflammatory disease. Although no effective means are available to prevent ovarian follicle decline, we hypothesize that oxidative stress, which is implicated in other aging processes, may also affect reproductive health. So antioxidant therapy, to control oxidant stress, may reduce the rate of reduction of ovarian follicles.

\section{b. Melatonin}

Melatonin is a neuroendocrine hormone secreted by the pineal gland. Secretion is regulated by light-and-dark stimuli ${ }^{8)}$, and the hormone influences circadian rhythm, such as the sleep-cycle and body temperature; melatonin also regulates other generative functions, such as sugar and lipid metabolism, carcinogenic control, immunity regulation ${ }^{9-12)}$, and reproduction.

In addition to the receptor mediated action as a neuroendocrine hormone, the discovery of melatonin as a direct free radical scavenger has greatly broadened the understanding of melatonin's mechanisms which benefit reproductive physiology ${ }^{13)}$. This cytoprotective action suggests that melatonin has potential as an Anti-Aging agent.

It has been believed that melatonin influences the hypothalamus - pituitary - gonadal axis and controls the reproductive function in seasonally breeding animals ${ }^{14)}$. However, recent discovery of gonadotropin inhibitory hormone $(\mathrm{GnIH})$ in birds showed that melatonin promotes production of $\mathrm{GnIH}$, which reduces the secretion of luteinizing hormone $(\mathrm{LH})$ and follicle-stimulating hormone $(\mathrm{FSH})^{14)}$. However, it is unclear whether melatonin suppresses ovarian function by GnIH secretion in human.

Melatonin may act directly on the ovaries. Several reports indicate high concentrations of melatonin in follicular liquid 15,16), and the fact that this concentration increases in proportion to ovarian follicle growth suggests that melatonin may influence ovulation ${ }^{17)}$. During oocyte maturation, the follicle grows until ovulation is induced by a surge in LH; this process produces reactive oxygen species (ROS) (Fig. 3). ROS play physiological roles in the process of ovulation, e.g. follicle rupture and oocyte maturation. However, an excessive amount of ROS cause oxidative stress and may damage oocyte and granulosa cells. Therefore, reduced oocyte quality and inhibited luteinization of granulosa cells (progesterone production) may be causes of infertility in women.

Analysis of follicular fluid from the women who underwent in vitro fertilization and embryo transfer (IVF-ET) found that melatonin concentrations increased depending on follicular growth. Intrafollicular concentrations of melatonin showed a negative correlation with 8-hydroxy-2'-deoxyguanosine (8-OHdG), as an oxidative stress marker. However, other antioxidants such as $\mathrm{Cu}, \mathrm{Zn}$-superoxide dismutase (SOD) and glutathione (GSH) did not show any correlation with 8-OHdG. It seems that melatonin is the most important antioxidant in the ovarian follicle ${ }^{18)}$.

Although oocyte maturation was inhibited by hydrogen peroxide $\left(\mathrm{H}_{2} \mathrm{O}_{2}\right)$, reduced oocyte maturation caused by $\mathrm{H}_{2} \mathrm{O}_{2}$ was recovered by the addition of melatonin in mouse oocyte incubation ${ }^{19,20)}$. Progesterone production by granulosa cells was also inhibited by $\mathrm{H}_{2} \mathrm{O}_{2}$ administration, and reduced progesterone production was recovered by melatonin administration in human granulosa cell culture ${ }^{18)}$.

Since melatonin may have a beneficial effect on oocyte maturation, it is possible that melatonin treatment can be a useful therapy for infertile women. Therefore, recently melatonin has been applied to infertility patients to improve oocyte quality ${ }^{19,20)}$. Patients who failed to become pregnant in the previous IVF-ET cycle with a low fertilization rate $(<$ $50 \%$ ) were given melatonin treatment in the next IVF-ET cycle. When patients were given a $3 \mathrm{mg}$ tablet of melatonin orally at 22:00 hr from the fifth day of the previous menstrual cycle until the day of oocyte retrieval, intra-follicular concentrations of melatonin were increased and $8-\mathrm{OHdG}$ were decreased compared to those in the previous cycle. In 56 patients who underwent melatonin treatment, the fertilization rate $(50.0 \pm 38.0 \%)$ was markedly improved compared with the
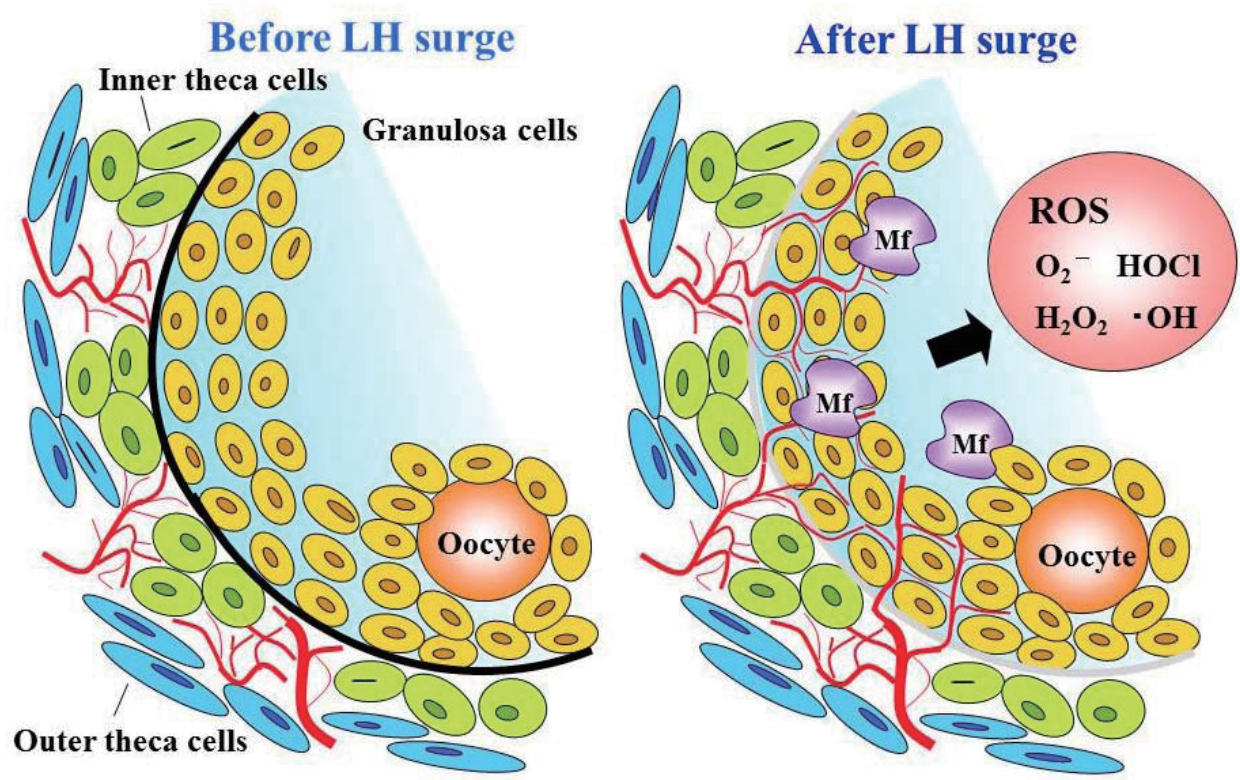

Fig. 3. Changes in the ovary after a surge in luteinizing hormone, showing the generation of reactive oxygen species. 
previous IVF-ET cycle $(20.2 \pm 19.0 \%)$, and 11 of 56 patients $(19.6 \%)$ achieved pregnancy. On the other hand, in 59 patients who were not given melatonin, the fertilization rate $(22.8 \pm 19.0 \%$ vs. $20.9 \pm 16.5 \%$ ) was not significantly changed, and only 6 of 59 patients $(10.2 \%)$ achieved pregnancy. These results show that melatonin administration increases intra-follicular melatonin concentrations, reduces intra-follicular oxidative damage and elevates fertilization and pregnancy rates.

Not only acute stress during ovulation processes but also chronic stress due to aging in women, such as physical and chemical stimuli, seem to be causes of poor oocyte quality and follicle loss (Fig. 4). Although there is currently no effective method to prevent decreases in oocyte quality and follicle loss, melatonin is the most promising treatment. Recent evidence demonstrated that decreased melatonin secretion due to aging was associated with age-related diseases, such as osteoporosis and Alzheimer's disease ${ }^{21,22)}$, and melatonin administration has been shown to prevent and/or ameliorate these diseases. Growing evidence suggests that melatonin is effective as an Anti-Aging supplement. In addition, other research also demonstrated that melatonin prevents the reduction in the number of ovarian follicles caused by immunologically induced stress with anti-ovary antibodies ${ }^{23)}$.

As summarized above, it is possible that melatonin treatment prevents ovarian aging, but further research is required to assess whether long-term melatonin treatment can prevent decreases in oocyte quality and follicle loss.

\section{c. Growth hormone and insulin growth factor-I}

Growth hormone $(\mathrm{GH})$ is secreted from the anterior pituitary gland in response to stimuli from sleep, movement, or meals. GH acts on the liver and triggers the production of a second messenger hormone, insulin-like growth factor-I (IGF-I) 1). Blood concentrations of GH and IGF-I start to fall from around age 30, with concentrations of these hormones proving indicative of health and QOL.

Ovarian maturity is controlled by the endocrine, autocrine, and paracrine signals, and IGF-I stimulates ovarian maturation in the same way as gonadotropin ${ }^{24-27)}$. GH and IGF-I trigger E2 and progesterone production from granulosa cells ${ }^{10,28)}$
Low IGF-I production at 20 to 30 years is one possible cause of ovary functional failure.

Several authors have reported that GH medication at the time of IVF transfer provides benefits to the patient ${ }^{29-32)}$. Tesarik et al. in a study of women (age $>40$ years) undergoing IVF, reported that GH increased the peak serum E2, ovarian follicle E2, fertility rate, and the birthrate ${ }^{29}$. A Japanese study is also performed in the poor gonadotropin responders to whom $\mathrm{GH}$ secretion was not induced after intravenous administration of growth hormone-releasing factor ${ }^{32)}$.

These data imply that the youth and health of the ovary may be maintained by improvements in lifestyle that prevent the eventual reduction in GH/IGF-I secretion. High-quality sleep, moderate exercise, a balanced diet containing protein and amino acids (e.g., arginine), and regular meals all promote ghrelin secretion, which in turn triggers GH secretion ${ }^{1)}$. In contrast, a lack of, or poor-quality, sleep caused by mental and physical stress, lack of exercise, or excessive consumption of carbohydrates may all inhibit GH secretion. Other conditions also alter hormone levels. An additional consideration is that the liver may inhibit the action of orally administered estrogen on IGF-I production; thus, the percutaneous estrogen administration may benefit GH/IGF-I secretion.

In polycystic ovary syndrome (PCOS), levels of several hormones change: fasting insulin increases (increase in insulin resistance), IGF binding protein (IGFBP) and sex hormone binding globulin (SHBG) decrease, androgen, and free IGF-I increase ${ }^{33)}$. Insulin resistance also increases with visceral fat accumulation, metabolic syndrome, and type 2 diabetes, and these conditions cause a compensatory rise in the IGF-I value. In these cases, a decrease in insulin resistance will decrease IGF-I levels, and the change in hormone levels may benefit the whole body balance.

\section{d. Dehydroepiandrosterone-sulfate}

Dehydroepiandrosterone-sulfate (DHEA-s) is the most abundant steroid hormone in the body and is a precursor of about 50 other hormones, including sex and protein anabolic hormones ${ }^{1)}$. The administration of DHEA improves immunity and stress resistance and helps prevent lifestyle-related diseases,

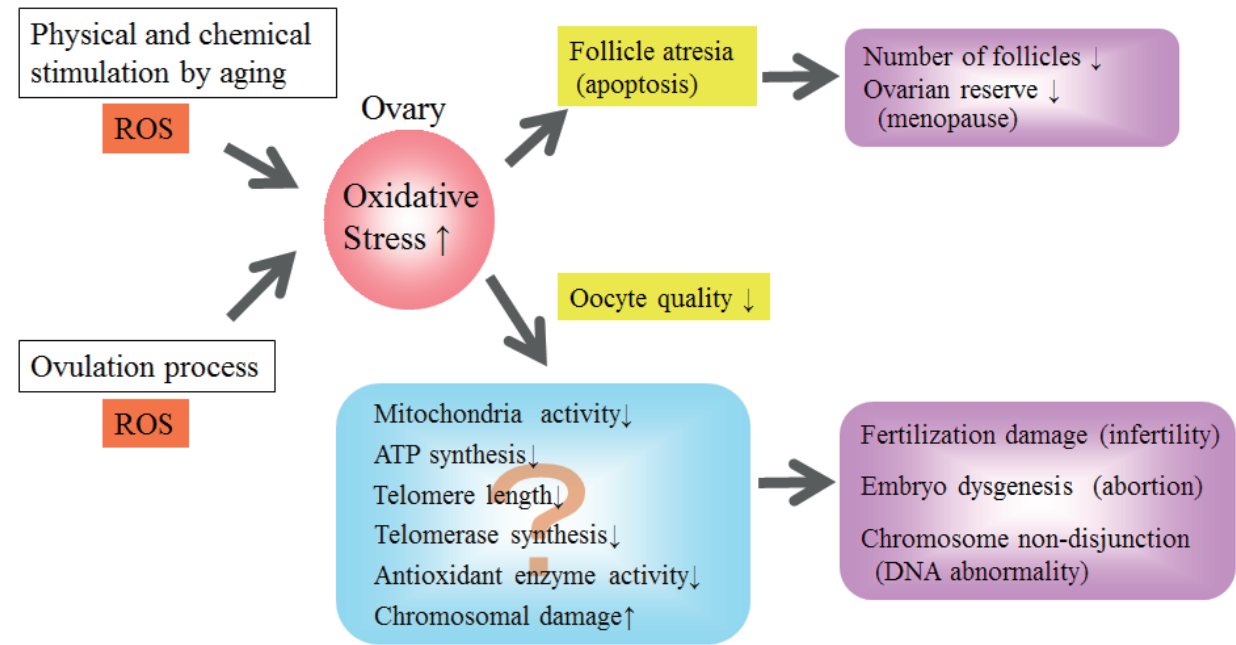

Fig. 4. Diagram demonstrating possible mechanisms of age-related ovary and oocyte injury. 
such as diabetes, hyperlipemia, hypertension, and osteoporosis. The blood concentration of DHEA-s decreases with age 34), and DHEA-s concentration is an important predictor of longevity ${ }^{35}$ ). Younger women (aged 20-30 years) who present with abnormally low blood concentrations of DHEA-s have a higher risk of ovarian failure than women with high or normal concentrations. Patients suffering from visceral fat storage, metabolic syndrome, or diabetes, tend to show increased insulin resistance and decreased DHEA-s secretion ${ }^{36)}$, which increases IGFBP and inhibits the action of IGF-I.

The administration of DHEA ( $75 \mathrm{mg} /$ day) for approximately 4 months to 25 patients undergoing IVF who were diagnosed with low ovarian levels of DHEA-s, increased the yield of oocytes and embryos, indicating ovary function was significantly improved ${ }^{37}$ ). Fertility clinics in Western countries often use DHEA during treatment; when the compound was prescribed to 19 women (poor responders or women over 38 years of age) in Japan being treated with assisted reproductive technology (ART), several measures of ovary function, such as peak E2 levels, improved significantly ${ }^{38)}$. A further trial of DHEA administration during ART found the yield of oocytes, fertilized ova, and peak E2 levels all increased ${ }^{39)}$.

\section{Muscle age}

For most people, muscle mass decreases by $1 \%$ per year from about age $25^{40}$ ). This reduction in muscle strength may subsequently reduce the QOL, which may be particularly important in women, as they generally have less muscle mass than men.

Skeletal muscles form the largest organ in the body, comprising approximately half the total weight. As approximately $70 \%$ of the intake of dietary glucose is absorbed by muscle, maintaining muscle mass may help to prevent glycation stress. Physical exercise, especially muscle training, also effects insulin metabolism. In the insulindependent pathway, insulin binds to an insulin receptor, glucose transporter-4 (GLUT4), and is transported through the cytoplasm and passed through the cell membrane ${ }^{41)}$. In the insulin-independent pathway, skeletal muscle contraction transports GLUT4 through adenosine monophosphate-activated protein kinase (AMPK). Muscle training effectively improves insulin resistance, which may reduce the risk of PCOS cited above.

\section{Bone age}

In both genders, bone density begins to decline from age $30^{1)}$, but in post menopausal women, decreasing estrogen levels increases the metabolic turnover in bone, thereby decreasing bone density to an even greater extent than in men. This phenomenon may also occur in younger women experimenting with extreme diets which may increase the loss of bone minerals and subsequently cause a rapid decrease in bone density.

\section{Vascular age}

While degeneration in the vascular system that commonly occurs with age ${ }^{1)}$ rarely presents a serious problem for people younger than ages 30 to 40 , vascular dysfunction may cause serious health problems for older persons. Arteriosclerosis derived from hypertension, diabetes, dyslipidemia, and smoking may have negative effects on ovarian function.

\section{Neural age}

Neural and mental functions decrease with age as the number of brain neurons falls ${ }^{1)}$, but this decline rarely causes serious problem for people aged 30 to 40 years or younger. Careful control of mental and bodily stress, as well as maintaining good quality sleep, help preserve neuronal function.

\section{Aging risk factors}

\section{Immune stress}

In most people, the effectiveness of the immune system, and thus defense against adventitious pathogens, deteriorates with age; however, DHEA-s promotes immune function and can therefore be used to evaluate immune function. Although reduced immune function is rare in younger women age 30 to 40 , the prevalence of some allergic diseases, such as hay fever, asthma, and atopic dermatitis, has increased in recent years among this particular subpopulation.

\section{Mental and physical stress}

The most useful index of mind-and-body stress is the serum DHEA-s/cortisol ratio. A ratio of $\geq 20$ is considered ideal, 13-20 is regarded as borderline, and $<13$ indicates overstress ${ }^{1)}$. The overstress condition adversely affects ovarian function, suppresses estrogen secretion, and causes menstrual disorders; in extreme cases, overstress may stop menstruation altogether. Stress control should be an important part of clinical practice for reproductive medicine.

\section{Oxidative stress}

Oxidative stress is a significant age-related risk factor which may damage ovarian function (Fig. 4) by reducing mitochondria activity ${ }^{42}$, thereby leading to cytoplasm deterioration and reduction in antioxidant potential. In the ovary, these changes impair the fertilization and development of oocytes ${ }^{43,44)}$, but such oxidative stress may be countered by consumption of antioxidants, such as vitamin E and Coenzyme Q10.

Mitochondrial dysfunction in oocytes may delay preimplantation embryonic development and inhibit embryonic growth during ART. One technique used to treat sterility is ooplasmic transfer, where mitochondria from younger donors are transplanted to older recipients ${ }^{45}$ ). After embryo implantation, ovarian hormonal secretion is maintained, and the activity of antioxidant enzymes such as $\mathrm{Cu}, \mathrm{Zn}-\mathrm{SOD}$ increases. This inhibits the accumulation of active oxygen in endometrial cytoplasm thus promoting a beneficial environment during pregnancy ${ }^{46)}$.

Advanced anti-oxidative medical treatment is more effective when treated by a combination of aerobic exercise, which enhances antioxidant potential, and consumption of antioxidants.

\section{Glycation Stress}

Glycation, or Maillard, reactions occur between proteins and reducing sugars (e.g., glucose, fructose). The reaction 
produces advanced glycation end products (AGEs) via intermediate compounds, such as Amadori products ${ }^{47)}$.

Protein glycation leads to the accumulation of AGEs, and the binding of AGEs to receptor for AGEs (RAGE) induces cytotoxicity. For example, excess intracellular glucose alters the TCA cycle and increases fumarate levels. Fumarate reacts with cysteine residues in proteins, forming (2-succinyl) cysteine (2SC). The addition of $2 \mathrm{SC}$ to various proteins, such as cytoskeletal proteins, heat shock proteins, and adiponectin, inhibits their proper function. These changes are called glycation stress ${ }^{48)}$.

Analysis of AGEs in plasma and follicular fluid samples shows that AGEs accumulation harms follicular growth, fertilization, embryo development, and pregnancy. Although these factors are independent, calendar age, day-3-FSH (serum FSH concentration measured on the third day of the menstrual cycle), and AGE accumulation may all indicate poor responders to IVF treatment ${ }^{49)}$

The administration of metformin, which is an anti-diabetic agent that decreases glucose production by the liver and lowers plasma glucose levels, may also reduce glycation stress and thus improve IVF success ${ }^{50)}$.

Results from a study comparing soluble RAGE levels in plasma between patients younger or older than 35 years of age showed that soluble RAGE levels were significantly higher in younger patients ${ }^{51)}$. The binding of AGEs to soluble RAGE, which acts as a decoy receptor, inhibits RAGE activation at the cell membrane ${ }^{47)}$. Thus, younger people have higher resistance to glycation stress.

Glycation stress may be reduced by: 1) a diet that prevents rapid increases in blood sugar and insulin levels (eating slowly, chewing properly, correct eating order $[i . e$. fiber rich foods, followed by protein, followed by carbohydrates], avoiding sweets, juice, and carbonated beverages) and 2) maintaining skeletal muscle mass ${ }^{48)}$. For those with high glycation stress caused by poor eating habits, diabetes, and metabolic syndrome, anti-glycation supplements ${ }^{52,53)}$ currently being developed may eventually be available. Further investigation is necessary to establish the effectiveness of anti-glycation supplements on IVF therapy.

\section{Lifestyle}

"Lifestyle" includes eating, exercise, sleeping, smoking and drinking habits, and fluid intake. Smoking and excessive drinking increase oxidative stress and degrade ovary function, and lack of sleep or irregular sleeping habits delay recovery from the damage caused by mind-and-body stress, and negatively influence ovarian function. Similarly, a poor diet also increases glycation stress. As a lack of exercise decreases ovary function, infertility consultants encourage exercise as part of a treatment.

When the effect of two exercise programs (low activity and walking) was evaluated in women aged approximately 40 years, serum IGF-I and DHEA-s increased by $17.0 \%-17.6 \%$ and $22.8 \%-34.2 \%$ respectively ${ }^{54)}$. This information is relevant to IVF programs, since the IVF success rate decreases around age 40 .

In patients where ovarian function has deteriorated to the point that IGF-I and DHEA-s are lower than the average for their age group, any improvement in hormonal secretion by $20 \%-30 \%$, is likely to benefit ovary function. Exercise treatment will improve the results for patients treated for reproductive difficulty.

\section{Conclusion}

Here, we suggest that Anti-Aging Medicine may be applicable to reproductive medicine. Effective reproductive medicine is not confined to reproductive organs, but should also consider the balance of the body. Anti-Aging medical indices that evaluate specific systems, such as vascular condition and age-related risk factors, provide a framework that enables clinical evaluation of "degradation of an ovary function" and "ovary insufficiency" for each patient. The poor response to IVF noted in some patients may be due to low levels of hormonal secretion, with factors such as mental, physical, oxidative, and glycation stress also potentially reducing ovary function. Given that ovary function varies by individual, type and degree of aging should be assessed on an individual basis to identify the primary risk factors. A combination of AntiAging Medicine and advanced medical technology may help to rejuvenate ovary function. We expect the implementation of Anti-Aging Medicine in therapeutic regimens to benefit reproductive medicine.

\section{Conflict of interest statement:}

The authors declare no financial or other conflicts of interest in the writing of this paper. 


\section{References}

1) Yonei Y: "Introduction to Anti-Aging Medicine," 2nd ed., Keio University Publication, Tokyo, 2011 (in Japanese)

2) Akasofu K, Shozu M: Menopause and endoclinological changes. Journal of Medical Technology 37; 349-354: 1993 (in Japanese)

3) Pelletier G: Localization of androgen and estrogen receptors in rat and primate tissues. Histol Histopathol 15; 1261-1270: 2000

4) Ikenoue N, Wakatsuki A, Okatani Y: Small low density lipoprotein particles in women with natural or surgically induced menopause. Obster Gynecol 93; 566-570: 1999

5) Mirza FS, Prestwood KM: Bone health and aging: implications for menopause. Endocrinol Metab Clin North Am 33; 741-759: 2004

6) Molina JR, Barton DL, Loprinzi CL: Chemotherapy-induced ovarian failure: manifestations and management. Drug Saf 28; 401-416: 2005

7) Cohen LE: Cancer treatment and the ovary: the effects of chemotherapy and radiation. Ann NY Acad Scl 1135; 123-125: 2008

8) Yonei Y, Hattori A, Tsutsui K, et al: Effects of melatonin: Basics studies and clinical applications. Anti-Aging Medicine 7; 85-91: 2010

9) Tamura H, Nakamura Y, Narimatsu A, et al: Melatonin treatment in peri- and postmenopausal women elevates serum highdensity lipoprotein cholesterol levels without influencing total cholesterol levels. J Pineal Res 45; 101-105: 2008

10) Korkmaz A, Tamura H, Manchester LC, et al: Combination of melatonin and a peroxisome proliferator-activated receptorgamma agonist induces apoptosis in a breast cancer cell line. J Pineal Res 46; 115-116: 2009

11) Reiter RJ, Tan DX, Korkmaz A, et al: Light at night, chronodisruption, melatonin suppression, and cancer risk: a review. Crit Rev Oncog 13; 303-328: 2007

12) Reiter RJ, Tan DX, Manchester LC, et al: Melatonin defeats neurally-derived free radicals and reduces the associated neuromorphological and neurobehavioral damage. J Physiol Pharmacol 58 (Suppl 6); 5-22: 2007

13) Tan DX, Manchester LC, Terron MP, et al: One molecule, many derivatives: a never-ending interaction of melatonin with reactive oxygen and nitrogen species? J Pineal Res 42; 28-42: 2007

14) Tsutsui K: A new key neurohormone controlling reproduction, gonadotropin-inhibitory hormone $(\mathrm{GnIH})$ : Biosynthesis, mode of action and functional significance. Progress in Neurobiology 88; 76-88: 2009

15) Brzezinski A, Seibel MM, Lynch HJ, et al: Melatonin in human preovulatory follicular fluid. J Clin Endocrinol Metab 64; 865867: 1987

16) Ronnberg L, Kauppila A, Leppaluoto J, et al: Circadian and seasonal variation in human preovulatory follicular fluid melatonin concentration. J Clin Endocrinol Metab 71; 492-496: 1990

17) Nakamura Y, Tamura H, Takayama H, et al: Increased endogenous level of melatonin in preovulatory human follicles does not directly influence progesterone production. Fertil Steril 80; 1012-1016: 2003

18) Taketani T, Tamura H, Takasaki A, et al: Protective role of melatonin in progesterone production by human luteal cells. J Pineal Res 51; 207-213: 2011

19) Tamura H, Nakamura Y, Korkmaz A, et al: Melatonin and the ovary; physiological and pathophysiological implications. Fertil Steril 92; 328-343: 2009

20) Tamura H, Takasaki A, Miwa I, et al: Oxidative stress impairs oocyte quality and melatonin protects oocytes from free radical damage and improves fertilization rate. J Pineal Res 44; 280-287: 2008

21) Suzuki N, Somei M, Seki A, et al: Novel bromomelatonin derivatives as potentially effective drugs to treat bone diseases. J Pineal Res 45; 229-234: 2008

22) Bubenik GA, Konturek SJ: Melatonin and aging: prospects for human treatment. J Physiol Pharmacol 62; 13-19: 2011
23) Voznesenskaya T, Makogon N, Bryzgina T, et al: Melatonin protects against experimental immune ovarian failure in mice. Reprod Biol 7; 207-220: 2007

24) Giudice LC: Insulin-like growth factors and ovarian follicular development. Endocr Rev 13; 641-669: 1992

25) Campbell BK, McNeilly AS: Follicular dominance and oocyte maturation. Zygote 4; 327-334: 1996

26) Gutiérrez CG, Campbell BK, Webb R: Development of a longterm bovine granulosa cell culture system: induction and maintenance of estradiol production, response to folliclestimulating hormone, and morphological characteristics. Biol Reprod 56; 608-616: 1997

27) Poretsky L, Cataldo NA, Rosenwaks Z, et al: The insulin-related ovarian regulatory system in health and disease. Endocr Rev 20; 535-582: 1999

28) Taketani T, Yamagata Y, Takasaki A, et al: Effects of growth hormone and insulin-like growth factor 1 on progesterone production in human luteinized granulosa cells. Fertil Steril 90; 744-748: 2008

29) Tesarik J, Hazout A, Mendoza C: Improvement of delivery and live birth rates after ICSI in women aged $>40$ years by ovarian co-stimulation with growth hormone. Hum Reprod 20; 25362541: 2005

30) Kucuk T, Kozinoglu H, Kaba A: Growth hormone co-treatment within a GnRH agonist long protocol in patients with poor ovarian response: a prospective, randomized, clinical trial. J Assist Reprod Genet 25; 123-127: 2008

31) Yovich JL, Stanger JD: Growth hormone supplementation improves implantation and pregnancy productivity rates for poor-prognosis patients undertaking IVF. Reprod Biomed Online 21; 37-49: 2010

32) Takasaki A: Ovarian poor responder in ART. Japanese Journal of Fertility and Sterility 47; 123: 2002 (in Japanese)

33) Iwashita M, Mimuro T, Watanabe $M$, et al: Plasma levels of insulin-like growth factor-I and its binding protein in polycystic ovary syndrome. Horm Res 33 (Suppl. 2); 21-26: 1990

34) Nomoto K, Arita S, Yonei Y: Development of a model of functional endocrine age in Japanese people: serum dehydroepiandrosterone-sulfate (DHEA-s) concentration as an index of aging. Anti-Aging Medicine 8; 69-74: 2011

35) Roth GS, Lane MA, Ingram DK, et al: Biomarkers of caloric restriction may predict longevity in humans. Science 297; 811: 2002

36) Sumida Y, Yonei Y, Kanemasa K, et al: Lower circulating levels of dehydroepiandrosterone, independent of insulin resistance, is an important determinant of severity of non-alcoholic steatohepatitis in Japanese patients. Hepatol Res 40; 901-910: 2010

37) Barad D, Gleicher N: Effect of dehydroepiandrosterone on oocyte and embryo yields, embryo grade and cell number in IVF. Hum Reprod 21; 2845-2849: 2006

38) Yoshida H, Aono N, Iwasa Y, et al: Effect of dehydroepiandrosterone (DHEA) on ovarian function in IVF cycles. Journal of Fertilization and Implantation (Tokyo) 25; 176179: 2008 (in Japanese)

39) Usui A, Kubo H: (Recent progress on ova) Medication for improvement of ovular quality: treatment with DHEA, supplementation for anti-aging. Obstetrical and Gynecological Therapy 96; 50-54: 2008 (in Japanese)

40) Yonei Y, Miwa Y, Hibino S, et al: Japanese anthropometric reference data: special emphasis on bioelectrical impedance analysis of muscle mass. Anti-Aging Medicine 5; 63-72: 2008

41) Hayashi T, Nakano M, Yonemitsu S: Development of a new exercise program based on the molecular mechanism of exercisestimulated glucose metabolism. Descente Sports Science 22; 3140: 2001 (in Japanese)

42) Naito Y, Lee CM, Kato Y, et al: Oxidative stress markers. AntiAging Medicine 7; 36-44: 2010 
43) Nagai S, Mabuchi T, Hirata S, et al: Correlation of abnormal mitochondrial distribution in mouse oocytes with reduced developmental competence. Tohoku J Exp Med 210; 137-144: 2006

44) Kanaya H, Hashimoto S, Teramura T, et al: Mitochondrial dysfunction of in vitro grown rabbit oocytes: results in preimplantation embryo arrest after activation. J Reprod Devlop 53; 631-637: 2007

45) Robertson JA: Ooplasmic transfer. N Engl J Med 347; 147-148: 2001

46) Sugino N: Role of superoxide radicals and superoxide dismutase in the regulation of human endometrial function. Acta Obstetrica et Gynaecologica Japonica 55; 1013-1023: 2003 (in Japanese)

47) Nagai R, Mori T, Yamamoto $Y$, et al: Significance of advanced glycation end products in aging-related disease. Anti-Aging Medicine 7; 112-119: 2010

48) Ichihashi M, Yagi M, Nomoto K, et al: Glycation stress and photo-aging in skin. Anti-Aging Medicine 8; 23-29: 2011

49) Jinno M, Takeuchi M, Watanabe A, et al: Advanced glycation end-products accumulation compromises embryonic development and achievement of pregnancy by assisted reproductive technology. Hum Reprod 26; 604-610: 2011
50) Jinno M, Kondou K, Teruya K: Low-dose metformin improves pregnancy rate in in vitro fertilization repeaters without polycystic ovary syndrome: Prediction of effectiveness by multiple parameters related to insulin resistance. Hormones 9; 161-170: 2010

51) Fujii E, NakayamaM: Concentrations of soluble RAGE, VEGF and AGEs in plasma and ovarian fluid of women with reproducible ages; effect of aging. The 10th meeting of Japanese Society of Anti-Aging Medicine, Kyoto: 2010 (abstract in Japanese)

52) Yonei Y, Miyazaki R, Takahashi Y, et al: Anti-glycation effect of mixed herbal extract in individuals with pre- diabetes mellitus: A double-blind, placebo-controlled, parallel group study. AntiAging Medicine 7; 26-35: 2010

53) Yagi M, Nomoto K, Hori M, et al: The effect of edible purple chrysanthemum extract on advanced glycation end products generation in skin: a randomized controlled clinical trial and in vitro study. Anti-Aging Medicine 9; 00-00: 2012 (in press)

54) Mochizuki T, Amenomori Y, Miyazaki R, et al: Evaluation of exercise programs at a fitness club in female exercise beginners using anti-aging medical indicators. Anti-Aging Medicine 6; 6678: 2009 